\title{
Using Feedback on Symptomatic Infections to Contain the Coronavirus Epidemic: Insight from a SPIR Model
}

\author{
Michael Nikolaou ${ }^{1}$ \\ ${ }^{1}$ Chemical \& Biomolecular Engineering Department, University of Houston, Houston, TX 77204-4004 \\ E-mail: nikolaou@uh.edu
}

\begin{abstract}
A study is presented on the use of real-time information about symptomatic infectious individuals to adjust restrictions of human contacts at two basic levels, the stricter being on the symptomatic infectious group. Explicit analytical formulas as well as numerical results are presented to rapidly elucidate what-if questions on averting resurgence of the coronavirus epidemic after the first wave wanes. Implementation of related ideas would rely on a mix of several factors, including personal initiative and sophisticated technology for monitoring and testing. For robust decision making on the subject, detailed multidisciplinary studies remain indispensable.
\end{abstract}

Keywords - COVID-19, Coronavirus, Flattening the Curve, Social Distancing, Population Dynamics, Control

\section{Introduction}

In the absence of a vaccine, restrictions on social distancing have been used widely in recent efforts to avert an inordinately large number of fatalities from potentially uncontrolled spread of COVID-19. ${ }^{1-3}$ Underlying these efforts are fundamental constraints emerging from the dynamics of infectious disease spreading, as captured in the most elementary form by the standard SIR model:4-7 The coronavirus basic reproductive ratio, $R_{0}$, naturally between 2 and 3 (Figure 1$)^{8}$ should be brought close to 1 (or below) by social distancing measures, ${ }^{8,9}$ with correspondingly flattened curves of percent-infectious over time. ${ }^{10-12}$

With the end of the first wave of the epidemic projected in a few weeks, questions arise about removal of social distancing measures. As desirable as the "return to normal" is, fundamental constraints remain that prevent complete removal of restriction measures until a vaccine is available for safe use. ${ }^{13}$ Indeed, the total fraction of infected - therefore eventually immune - by the end of the first wave will be at single percentage points, ${ }^{2}$ far below the $1-1 / R_{0} \approx 60 \%$ required to robustly avert resurgence of the epidemic. ${ }^{7}$ Therefore, a number of strategies are considered, such as alternating imposition and removal of measures over time ${ }^{1}$ stratification by age, pre-existing conditions, location, or other

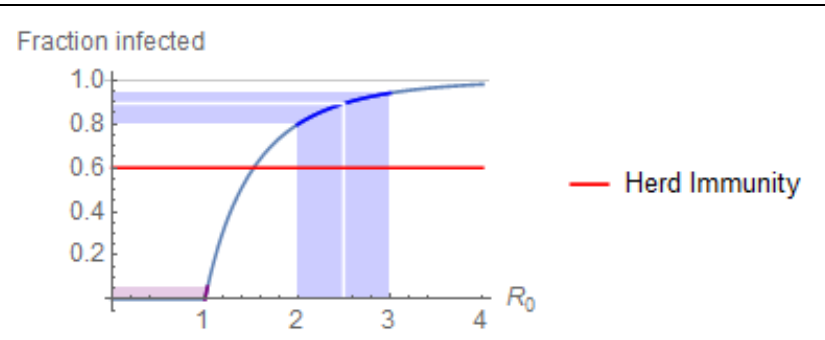

Figure 1. Total fraction of a population infected, $r(\infty)$ - thus rendered immune - by the end of an epidemic, as a function of the basic reproductive ratio $R_{0}$, according to the solution $r(\infty)=\frac{1}{R_{0}} \ln (1-r(\infty)) \Leftrightarrow r(\infty)=1-\frac{W\left[R_{0} \exp \left(R_{0}\right)\right]}{R_{0}}$ of the SIR model (similar to eqn. (9)). For $R_{0}<1$ the virus does not spread and the epidemic is contained. The infectious fraction reduces over time when the population fraction susceptible to infection is below $1-1 / R_{0} \approx 60 \%$ for the coronavirus.

risk-factors, ${ }^{1,14}$ mass screening, contact tracing, testing of all individuals entering the country, and quarantine of people who test positive. ${ }^{15}$

The purpose of this paper is to analyze the dynamics of simply placing restrictions on people infected by the virus after they have shown symptoms of the disease. No testing is 
medRxiv preprint doi: https://doi.org/10.1101/2020.04.14.20065698; this version posted April 28, 2020. The copyright holder for this preprint (which was not certified by peer review) is the author/funder, who has granted medRxiv a license to display the preprint in perpetuity. It is made available under a CC-BY-NC-ND 4.0 International license .

required during an individual's pre-symptomatic period, although such testing would certainly be beneficial, as that pre-symptomatic individual would be infective immediately upon infection. ${ }^{16}$ Restrictions may be self-imposed and assisted by telecommunications technology, a practice already implemented in Asian countries and under consideration in the US. ${ }^{17}$

In the rest of the paper, the basic analytical results are derived and illustrated by numerical simulations.

\section{Containing virus spread by monitoring symptoms}

The SPIR model structure (Figure 2) comprises population fractions susceptible to the virus, $s$; pre-symptomatic infectious, $p$; symptomatic infectious, $i$; and removed from the infectious pool, $r$, by recovery or death. A distinction between the SPIR model structure and other fourcompartment structures, such as the classic SEIR, ${ }^{4-7,18}$ is that individuals with coronavirus infection entering the $\mathrm{P}$ group can infect before symptoms appear. Therefore, it is practically formidable to monitor those infecting during the presymptomatic period, as this would require inordinately massive testing or widespread tracking of contacts to guide selective testing. On the other hand, monitoring individuals with symptoms is more reasonable, as symptoms are fairly characteristic of the infection and testing for confirmation of the infection can be highly targeted. ${ }^{19}$ This type of interaction between the S, I, and P compartments is captured in the model structure shown in Figure 2. This structure indicates dynamics by separately adjustable feedback from the I group, in addition to feedback from the P group typically considered.

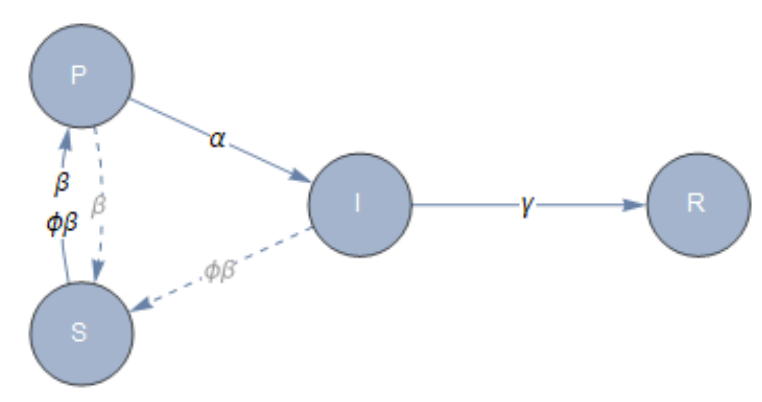

Figure 2. The SPIR model structure, with the pre-symptomatic infectious group, $\mathrm{P}$, and the symptomatic infectious group, I, infecting the susceptible group, $\mathrm{S}$, at different rates, due to different distancing measures.

Consequently, the following equations capture the dynamics of a fixed-size population with measures restricting transmission of the virus between susceptible and symptomatic infectious individuals:

$$
\begin{gathered}
\frac{d s}{d t}=-\phi \beta s i-\beta s p \\
\frac{d p}{d t}=\phi \beta s i+\beta s p-\alpha p
\end{gathered}
$$

$$
\frac{d i}{d t}=\alpha p-\gamma i
$$

where $\beta$ is the infection spread rate; $0 \leq \phi<1$ is a factor producing a reduced spread rate, $\phi \beta$, between susceptible and symptomatic infectious; $\alpha, \gamma$ are the removal rates from the pre-symptomatic and symptomatic infectious groups, respectively; and $R_{0} \stackrel{\text { def }}{=} \beta / \gamma$ is the basic reproductive ratio. The fourth fraction, $r=1-s-p-i$, refers to individuals removed from the infectious group, by either recovery or death. Clearly, then,

$$
\frac{d r}{d t}=\gamma i
$$

Based on eqns. (1)-(4) it can be easily shown (Appendix A) that herd immunity is achieved when the susceptible fraction, $s$, is less than

$$
S_{0}=\frac{1}{\beta\left(\frac{1}{\alpha}+\frac{1}{\gamma} \phi\right)}
$$

with obvious increase of $s_{0}$ towards $\alpha / \beta$ as $\phi \rightarrow 0$.

If no additional measures are taken to mitigate virus transmission from symptomatic infectious to susceptible, i.e. $\phi=1$, the resulting $s_{0}$ becomes

$$
S_{0}=\frac{1}{\beta\left(\frac{1}{\alpha}+\frac{1}{\gamma}\right)} \stackrel{\text { def }}{=} \frac{1}{R_{0}}
$$

where $\frac{1}{\alpha}+\frac{1}{\gamma}$ refers to the average time from becoming infected to removal from the infected group. Eqn. (6) implies that to keep $s_{0}=1-x \approx 1$, uniform restrictions on virus transmission must achieve

$$
R_{0}=\frac{1}{1-x} \approx 1+x \Rightarrow \beta_{\phi=1} \approx \frac{1+x}{\frac{1}{\alpha}+\frac{1}{\gamma}}
$$

However, with additional measures taken to mitigate virus transmission from symptomatic infectious to susceptible, i.e. $0 \leq \phi=1$, eqn. (6) implies that the resulting $\beta$ becomes

$$
\beta_{\phi<1}=\beta_{\phi=1} \frac{\frac{1}{\alpha}+\frac{1}{\gamma}}{\frac{1}{\alpha}+\frac{1}{\gamma} \phi}=\beta_{\phi=1} O\left(\frac{2}{1+\phi}\right)
$$

assuming $\alpha$ and $\gamma$ are of the same order of magnitude. ${ }^{20}$ In the extreme, if unrealistic, case of perfect detection and full quarantine for the symptomatic infectious $(\phi=0)$, restrictions on the rest of the population would be about half the size of equivalent uniform restrictions on the entire population $(\phi=1)$.

It should be noted that eqns. (1)-(4) suggest that even perfect restriction of virus transmission from the symptomatic infectious alone cannot yield adequate results, as the presymptomatic infectious would be enough to infect the population at numbers not lower enough than those without any restrictions. Indeed, it can be shown (Appendix $B$ ) that the total fraction of the population infected by the end of the epidemic, $r(\infty)$, satisfies the equation 
medRxiv preprint doi: https://doi.org/10.1101/2020.04.14.20065698; this version posted April 28, 2020. The copyright holder for this preprint (which was not certified by peer review) is the author/funder, who has granted medRxiv a license to display the preprint in perpetuity.

$$
\begin{gathered}
1-r(\infty)=\exp [-(\alpha / \beta) r(\infty)] \Leftrightarrow \\
r(\infty)=1-W[-\beta / \alpha \exp (-\beta / \alpha)] /(\beta / \alpha)
\end{gathered}
$$

(where $W$ is the Lambert function ${ }^{21,22}$ )* with

$$
\frac{\beta}{\alpha}=R_{0} \frac{1}{1+\alpha / \gamma} \approx \frac{1}{2} R_{0}
$$

for $\alpha \approx \gamma$, which in turn yields a total fraction of infected by the end of the epidemic around 60\% (Figure 1). Therefore coordinated reduction of both $\phi$ and $\beta$ (restrictions both for the symptomatic infectious and for the rest of the population, respectively) would be required for desirable results.

Given a growing body of data that can be used to estimate the effect of corresponding policies on the resulting $\beta,{ }^{9,23}$ the above simple analysis can help gauge restriction measures at the early conceptual level of making structural decisions.

\section{Simulations}

The values $\alpha=\frac{1}{5.1}$ days, $\gamma=\frac{1}{3.3}$ days, $R_{0}=2.5$ are used in all simulations. ${ }^{20}$ The well known basic case of doing nothing to contain the virus is shown in Figure 3, for reference.

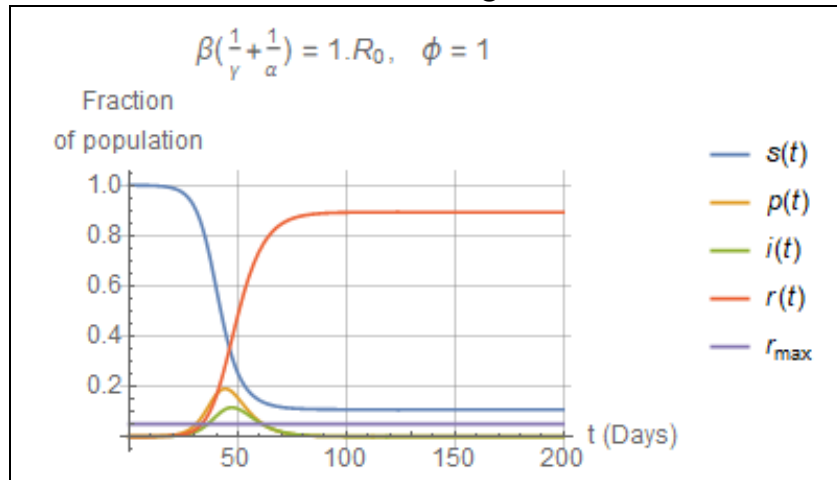

Figure 3. Basic case of progress of the coronavirus epidemic in the absence of any restrictions on the population. The cumulative fraction of infected in the epidemic, equal to $r(\infty)$, would be about 0.9, in agreement with Figure 1 for $R_{0}=2.5$.

In the next case, the symptomatic infectious part of the population is placed on quarantine, $\phi=0$ (Figure 4).

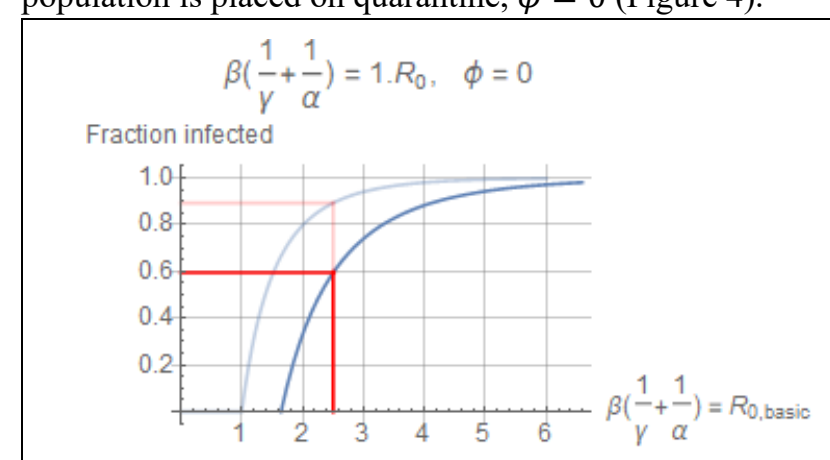

Figure 4. Placing the symptomatic infectious on quarantine without placing any restrictions on the rest of the population would yield $r(\infty)=1-W[-(\beta / \alpha) \exp (-\beta / \alpha)] /(\beta / \alpha)=0.6$. The curve shown in Figure 1 is also included for comparison.
The final value $r(\infty)$ suggested by eqns. (9) and (10), shown in Figure 4, is confirmed by the profile of $r(t)$ shown in Figure 5. The improvement over Figure 3 is inadequate.

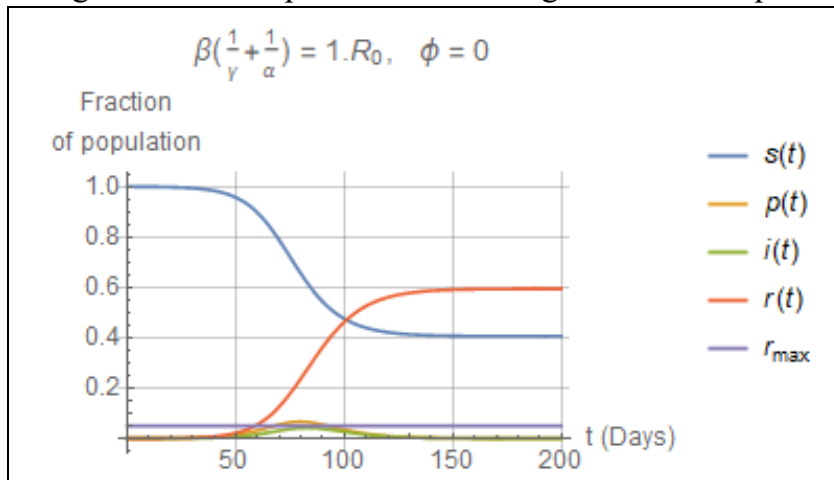

Figure 5. Placing the symptomatic infectious on quarantine would yield $r(\infty)=1-W[-(\beta / \alpha) \exp (-\beta / \alpha)] /(\beta / \alpha)=0.6$ as suggested by Figure 4.

Finally, placing restrictions on both the symptomatic infectious and the rest $\left(0.6 R_{0}\right)$ with tighter restrictions on the former $(\phi=0.3)$ yields quite improved results (Figure 6).

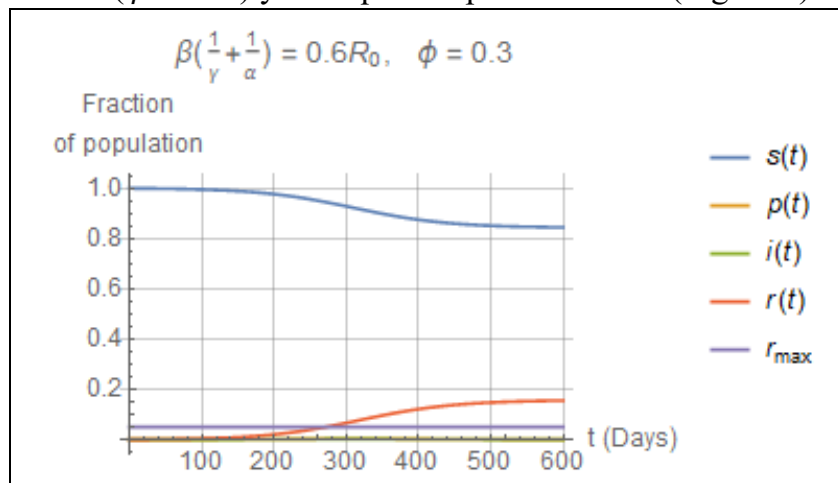

Figure 6. Placing restrictions on both the symptomatic infectious and the rest of the population with tighter restrictions on the former yields significantly improved results.

Note the quite inferior outcomes, were tighter restrictions not placed on symptomatic infectious $(\phi=1$, Figure 7).

Fraction

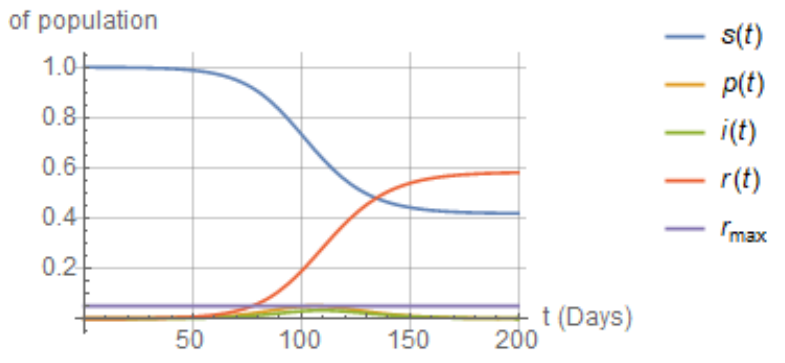

Figure 7. Not placing restrictions on the symptomatic infectious while leaving the rest of the population at the same restriction level yields far inferior outcomes. 
medRxiv preprint doi: https://doi.org/10.1101/2020.04.14.20065698; this version posted April 28, 2020. The copyright holder for this preprint

(which was not certified by peer review) is the author/funder, who has granted medRxiv a license to display the preprint in perpetuity.

It is made available under a CC-BY-NC-ND 4.0 International license .

\section{Discussion}

A study was presented on the use of real-time information about symptomatic infectious individuals to adjust restrictions of human contacts in areas affected by coronavirus infections. To the extent that measures can be taken to effectively implement such restrictions, they could help effective containment of the epidemic, particularly if combined with ideas tailoring measures to account for risk stratification. As already pointed out, successful implementation would hinge on a mix of several factors, including personal initiative and sophisticated technology for monitoring and testing. The simple formulas and graphs presented here, based on widely available background, will hopefully help provide guidelines for rapidly addressing what-if questions in a transparent way. For robust decision making detailed multidisciplinary studies remain indispensable. ${ }^{24}$

\section{Acknowledgements}

All computations were done in Mathematica, available at the University of Houston. Sharing of teaching material about the SIR model on Github by Prof. Jeff Kantor of Notre Dame is also gratefully acknowledged.

\section{References}

1 Ferguson, N. M. et al. Report 9 - Impact of nonpharmaceutical interventions (NPIs) to reduce COVID-19 mortality and healthcare demand. (Imperial College, https://doi.org/10.25561/77482, 2020).

2 Institute for Health Metrics and Evaluation (IHME). COVID-19 projections assuming full social distancing through May 2020, <COVID-19 projections assuming full social distancing through May 2020> (2020).

3 New York Times - Sheri Fink. Worst-Case Estimates for U.S. Coronavirus Deaths, $<$ https://nyti.ms/2w1vRKE $>$ (2020).

4 Kermack, W. O. \& McKendrick, A. G. A Contribution to the Mathematical Theory of Epidemics. Proceedings of the Royal Society of London Series A 115, 700 (1927).

5 Murray, J. D. Mathematical Biology: I. An Introduction. (Springer, 2002).

6 Anderson, R. M., Anderson, B. \& May, R. M. Infectious diseases of humans: dynamics and control. (Oxford University Press, 1992).

7 Keeling, M. J. \& Rohani, P. Modeling Infectious Diseases in Humans and Animals. (Princeton University Press, 2008).

8 Kucharski, A. J. et al. Early dynamics of transmission and control of COVID-19: a mathematical modelling study. The Lancet Infectious Diseases, doi:10.1016/S14733099(20)30144-4.

9 Wang, C. et al. Evolving Epidemiology and Impact of Non-pharmaceutical Interventions on the Outbreak of Coronavirus Disease 2019 in Wuhan, China. medRxiv, 2020.2003.2003.20030593, doi:10.1101/2020.03.03.20030593 (2020).
10 Murray, C. J. L. Forecasting COVID-19 impact on hospital bed-days, ICU-days, ventilator-days and deaths by US state in the next 4 months. medRxiv, 2020.2003.2027.20043752, doi:10.1101/2020.03.27.20043752 (2020).

11 Johns Hopkins Coronavirus Resource Center. Data Center, $<$ https://coronavirus.jhu.edu/data/new-cases $>$ (2020).

12 Dong, E., Du, H. \& Gardner, L. An interactive web-based dashboard to track COVID-19 in real time. The Lancet Infectious Diseases, doi:10.1016/S1473-3099(20)301201.

13 Leung, K., Wu, J. T., Liu, D. \& Leung, G. M. First-wave COVID-19 transmissibility and severity in China outside Hubei after control measures, and second-wave scenario planning: a modelling impact assessment. The Lancet, doi:10.1016/S0140-6736(20)30746-7.

14 Nikolaou, M. Simple Formulas for a Two-Tier Strategy to Flatten the Curve. arXiv.org, 2004.04271 (2020).

15 Institute for Health Metrics and Evaluation (IHME). IHME COVID-19 model FAQs. (2020).

16 Zhang, J. et al. Evolving epidemiology and transmission dynamics of coronavirus disease 2019 outside Hubei province, China: a descriptive and modelling study. The Lancet Infectious Diseases, doi:10.1016/S14733099(20)30230-9.

17 New York Times - Jack Nicas \& Daisuke Wakabayashi. Apple and Google Team Up to 'Contact Trace' the Coronavirus, $<$ https://nyti.ms/2UZrYji> (2020).

18 Blackwood, J. C. \& Childs, L. M. An introduction to compartmental modeling for the budding infectious disease modeler. Letters in Biomathematics 5, 195-221, doi:10.1080/23737867.2018.1509026 (2018).

19 Petherick, A. Developing antibody tests for SARS-CoV2. The Lancet 395, 1101-1102, doi:10.1016/S01406736(20)30788-1 (2020).

20 P, B. et al. Risk Assessment of Novel Coronavirus COVID-19 Outbreaks Outside China. Journal of Clinical Medicine 9 (2020).

21 Corless, R. M., Gonnet, G. H., Hare, D. E. G., Jeffrey, D. J. \& Knuth, D. E. On the LambertW function. Advances in Computational Mathematics 5, 329-359, doi:10.1007/BF02124750 (1996).

22 Kesisoglou, I., Singh, G. \& Nikolaou, M. The Lambert function belongs in the Function Hall of Fame. Chemical Engineering Education In preparation (2020).

$23 \mathrm{Koo}, \mathrm{J}$. R. et al. Interventions to mitigate early spread of SARS-CoV-2 in Singapore: a modelling study. The Lancet Infectious Diseases, doi:10.1016/S1473. 3099(20)30162-6.

24 Kissler, S. M., Tedijanto, C., Goldstein, E., Grad, Y. H. \& Lipsitch, M. Projecting the transmission dynamics of SARS-CoV-2 through the postpandemic period. Science, eabb5793, doi:10.1126/science.abb5793 (2020).

\footnotetext{
* Interestingly, the analytical solution for $r(\infty)$ in terms of the Lambert function $W(z)$, eqn. (9), pointed out as early as 1996, ${ }^{21}$ may have escaped the attention of most literature in this field. ${ }^{7}$
} 
medRxiv preprint doi: https://doi.org/10.1101/2020.04.14.20065698; this version posted April 28, 2020. The copyright holder for this preprint (which was not certified by peer review) is the author/funder, who has granted medRxiv a license to display the preprint in perpetuity.

It is made available under a CC-BY-NC-ND 4.0 International license.

Appendix A. Proof of eqn. (5)

Eqns. (2) and (3) imply

$$
\left[\begin{array}{l}
d e / d t \\
d i / d t
\end{array}\right]=\underbrace{\left[\begin{array}{cc}
\beta s-\alpha & \phi \beta s \\
\alpha & -\gamma
\end{array}\right]}_{\mathbf{A}}\left[\begin{array}{l}
e \\
i
\end{array}\right]
$$

The eigenvalues of the matrix $\mathbf{A}$ are in the left half-plane iff

$$
\operatorname{det}(\mathbf{A})>0
$$

and

$$
\operatorname{trace}(\mathbf{A})<0 \text {. }
$$

Eqn. (12) implies

$$
S<\frac{1}{\beta\left(\frac{1}{\alpha}+\frac{1}{\gamma} \phi\right)}
$$

Eqn. (13) implies

$$
s<\frac{\alpha+\gamma}{\beta}
$$

which is trivially guaranteed by eqn. (14), leading to eqn. (5).

Appendix B. Proof of eqn. (9)

Standard analysis of eqns. (1) and (2) for $\phi=0$ proceeds as follows:

$$
\begin{aligned}
& \frac{d s}{d e}=-\frac{\beta s}{\beta s-\alpha} \Rightarrow e=1-s+\frac{\alpha}{\beta} \ln s \Rightarrow \\
& 1-s(\infty)+\frac{\alpha}{\beta} \ln s(\infty)=0 \Leftrightarrow \\
& -\frac{\beta}{\alpha} e^{-\frac{\beta}{\alpha}}=\underbrace{-\frac{\beta}{\alpha} s(\infty)}_{Z} \exp \underbrace{\left[-\frac{\beta}{\alpha} s(\infty)\right]}_{Z} \Leftrightarrow \\
& -\frac{\beta}{\alpha} s(\infty)=W\left[-\frac{\beta}{\alpha} \exp \left(-\frac{\beta}{\alpha}\right)\right] \Rightarrow \\
& r(\infty)=1-s(\infty)=1+\frac{W\left[-\frac{\beta}{\alpha} \exp \left(-\frac{\beta}{\alpha}\right)\right]}{\frac{\beta}{\alpha}}
\end{aligned}
$$

